\title{
El automanejo de enfermedades crónicas: población de una jurisdicción de centros de salud
}

\author{
Peñarrieta-De Córdova María ${ }^{1}$, Vergel-Camacho Susana² ${ }^{2}$ Lezama-Vigo Sonia², \\ Rivero- Álvarez Rosario ${ }^{3}$, Taipe-Cancho Jorge ${ }^{4}$, Borda-Olivas Hilda ${ }^{5}$
}

\section{RESUMEN}

Objetivo: describir el automanejo en personas con enfermedades crónicas (diabetes, hipertensión y cáncer). Métodos: el estudio se realizó en poblaciones que pertenecían a la jurisdicción de centros de salud del Ministerio de salud, se realizó una muestra por conveniencias con el siguiente criterio de inclusión: mayores de 18 años y más de tres meses de presentar la enfermedad. Se aplicó el instrumento: «Automanejo en padecimientos crónicos. Partners in Health Scale» a un total de 382 personas. Resultados: el automanejo es deficiente, con una media general de 66, para diabetes, hipertensión y cáncer. Se encontró diferencias en el automanejo en la dimensión de adherencia así como de manejo de signos y síntomas. Las personas con diabetes presentaron mejor automanejo en el manejo de signos y síntomas frente a las personas con hipertensión y cáncer, mientras que las personas con cáncer presentaron mejor automanejo en adherencia comparadas con las personas con hipertensión y diabetes. Se encontró diferencias en el automanejo según sexo. Conclusiones: el automanejo en personas con enfermedades crónicas es deficiente en todas sus dimensiones: conocimiento, adherencia, y manejo de signos y síntomas. Se requiere realizar mayores investigaciones con perspectiva de género.

Palabras clave: enfermedades crónicas, autocuidado. Perú. (Fuente DeCs BIREME).

\section{The self-management of the chronic diseases: population of a jurisdiction of health centers}

\begin{abstract}
Objetive: To discover the self-management in people with chronic diseases (diabetes, hypertension and cancer). Methods: The research took place in population that belonged to the jurisdiction of health centers of the Ministry of Health, making a simple of conveniences with the following inclusion criteria: people over 18 years old, more than three months of having the disease. The instrument used was: «self-management of chronic suffering. Partners in Health Scale». A total of 382 people. Results: The self-management is deficient, with a general average of 66, for diabetes, hypertension and cancer. Differences were found in the self-management of the adherence dimension and the management of signs and symptoms. People with diabetes had better self-management in comparison with the people with hypertension and cancer; and people with cancer had better self-management in comparison with people with

Doctora en Ciencias de Salud Pública. Facultad de Enfermería, Universidad Autónoma de Tamaulipas, Tamaulipas, México.

Licenciada en Enfermería. Escuela de Enfermería, Universidad César Vallejo, Lima, Perú.

Magister en Enfermería. Escuela de Enfermería, Universidad César Vallejo, Lima, Perú.

Licenciada en Enfermería. Escuela de Enfermería, Universidad César Vallejo, Lima, Perú.

Candidata al Doctorado en Enfermería. Escuela de Enfermería, Universidad Cesar Vallejo, Lima, Perú.
\end{abstract}


hypertension and diabetes. Differences were found in the self-management according to gender. Conclusions. The self-management in people with chronic diseases is deficient, in all its dimensions: knowledge, adherence and management of signs and symptoms. More researches with gender perspective are needed.

Key words: Chronic disease self-management. Peru (Source DeCs BIREME).

\section{INTRODUCCIÓN}

Recientemente el tema de las enfermedades crónicas no transmisibles, entre las que se consideran a las enfermedades cardiovasculares, la diabetes, el sobrepeso y obesidad, el cáncer, y las enfermedades pulmonares crónicas ha alcanzado notoriedad mundial $(1,2)$. Hasta hace poco, las enfermedades crónicas no transmisibles que se observaban en países en desarrollo solían llamárseles «enfermedades de países ricos», sin embargo, hoy se sabe que el $80 \%$ del total de muertes a nivel mundial por estas causas ocurren en países de ingresos medios y bajos $(3,4)$. La adhesión al tratamiento apenas alcanza el 20\%, lo cual conduce hacia estadísticas negativas al área de salud, y ocasiona consecuencias perjudiciales para la familia, la sociedad y el gobierno (5). Las enfermedades crónicas no transmisibles (ECNT) son, pues, uno de los mayores retos que enfrenta el sistema de salud. Lo son por varios factores: el gran número de casos afectados; su creciente contribución a la mortalidad general; la conformación en la causa más frecuente de incapacidad prematura, y la complejidad y costo elevado de su tratamiento (5).

Este contexto genera enormes retos para la salud pública de muchos países, en donde las tareas y la agenda vinculada con enfermedades infecciosas y de la infancia no han concluido. Tal es el caso de Perú, donde el sistema de salud tiene que lidiar con el incremento de las enfermedades crónicas y, por otro lado, las infecciosas y de la infancia. Estudios recientes, dan cuenta de este incremento de enfermedades crónicas en el Perú. La prevalencia de diabetes fue $0,8 \%$ en zona rural, y tuvo un patrón de incremento en gradiente, $3 \%$ en migrantes y $6 \%$ en población urbana. Los estimados correspondientes en cada grupo para obesidad, también en un patrón de gradiente, fueron $3 ; 20$ y 33\%. La hipertensión arterial presenta alrededor del 11 al 15\%, mientras que es el doble, casi $30 \%$, en la población urbana; estas diferencias fueron notorias también según sexo en el grupo de migrantes (6). Es reconocido por el Ministerio de Salud como un problema de salud pública y se identifica, al menos, cuatro enfermedades crónicas prioritarias: hipertensión, diabetes, obesidad y cáncer (7).
Así mismo, algunos estudios muestran problemas relacionados con el conocimiento insuficiente de la enfermedad crónica y su manejo, como es el caso de las personas con diabetes (8). Otro estudio mostró que el nivel de conocimiento adecuado no estaba relacionado con el control glucémico y que el conocimiento es apenas una de las variables que puede influenciar en el control metabólico; así también menciona que el estilo de vida y las creencias pueden también tener gran impacto en el comportamiento de las personas (9).

Estudios realizados en el Perú (10) muestran que el 54,7\% presentó un nivel de conocimiento medio sobre el autocuidado en la prevención de complicaciones diabéticas, el 30,0 \% presentó un nivel de conocimiento bajo, mientras que el 15,3\% tuvo un nivel de conocimiento alto. Baca Martínez, B. et al., clasificó el conocimiento en suficiente, regular e insuficiente y refleja los siguientes resultados: insuficiente 78,7\%; regular $15,9 \%$, y suficiente 5,4\% de los 94 usuarios entrevistados (11). Así mismo, Corbacho et al. encontraron un nivel bueno con $23,0 \%$ (40); regular 37,9\% (66), y malo39,1\% (68) (12). A pesar que aún son muy pocos los estudios que evalúan el comportamiento de las personas con enfermedad crónica en sus cuidados, al menos en Perú, estos resultados caracterizan una problemática que merece destacar y que refuerza la necesidad de buscar estrategias innovadoras para promover la adhesión de esa población al tratamiento instituido, así como conseguir una participación mayor en sus propios cuidados. Algunas estrategias para este tipo de población incluyen las teorías de modelo de creencias en salud, autoeficacia, locus de control, disonancia cognitiva, difusión, pasantías de aprendizaje y, finalmente, la teoría de adultos (13). En el presente estudio se ha adoptado el modelo de colaboración, donde el corazón de este modelo es promover el automanejo.

Esta es una visión que difiere a la visión tradicional, que está centrada en la enfermedad, lo cual ha sido demostrado por muchos autores, esta es una visión que resulta insuficiente y, con frecuencia, un obstáculo para profundizar en otras facetas de la problemática que desafía a los servicios de salud, donde se señala que en multitud de 
enfermedades crónicas, lo cultural suele tener una influencia decisiva en su devenir, pero el predominio del enfoque nosológico como la aproximación casi exclusiva del médico en la atención de pacientes, obstaculiza o impide tomar conciencia de todo lo que ocurre fuera de dicho enfoque. Basado en este enfoque (tradicional) se realiza muchos programas educativos que se centra en informar a la persona sobre su enfermedad, en vez de una educación centrada en el automanejo (14). Esta educación (centrada en el automanejo) permite a los pacientes identificar sus problemas y entrega técnicas que los ayudan a tomar decisiones, tomar acciones apropiadas y cambiar estas acciones si ven un cambio en sus circunstancias o en la enfermedad. La educación en el automanejo complementa en vez de sustituir la educación tradicional. Es central un plan de acción de corto plazo de una a dos semanas y en el cual los pacientes tengan la confianza que puedan cumplirlo. Otro aspecto es aumentar la confianza del paciente de que puede lograr un plan que debe ser acordado colaborativamente entre el paciente y el médico u otro profesional de salud.

El cuidado colaborativo y la educación del automanejo tienen una unidad conceptual. Ambos tienen énfasis en el paciente como principal cuidador, aunque queda una gran responsabilidad en los profesionales de la salud quienes deben usar su experiencia para informar, activar y ayudar a los pacientes en el automanejo de su condición. Es en este contexto que los aspectos de la relación médico-paciente, tales como la comunicación y la empatía, son esenciales para la disponibilidad del paciente y su habilidad para completar las actividades de automanejo (15-18).

Las evaluaciones de programas de automanejo han demostrado mejorar el comportamiento de las personas, así como la comunicación con los médicos y el estado de salud $(19,20)$, se detecta un mejor estado de salud, menor uso de los servicios de salud y menores complicaciones. Es así que el automanejo de condiciones crónicas ha sido visto como una estrategia viable para llenar el vacío existente entre la capacidad del individuo y del sistema de salud para satisfacer las necesidades de los individuos con enfermedades crónicas (21). Comprender las relaciones entre las características de los pacientes, activación, automanejo y los resultados en la salud deseados es un importante próximo paso. Se necesitan mayores estudios para informar a los tomadores de decisiones sobre la implementación de programas de automanejo para las enfermedades crónicas, aspecto que se pretende cubrir, al menos, en una primera etapa en el presente proyecto, con la descripción del automanejo en personas con enfermedades crónicas en una población de Lima, Perú.

\section{MATERIAL Y MÉTODOS}

El estudio corresponde a la primera fase de un proyecto binacional (Escuela de Enfermería, Universidad Cesar Vallejo, Lima -Perú y La Facultad de Enfermería, Tampico, Universidad Autónoma de Tamaulipas, México), concierne a la fase de diagnóstico del automanejo en personas con enfermedades crónicas (hipertensión, diabetes y cáncer). La población estudiada es resultado de una muestra por conveniencia. Se seleccionaron a las personas que refirieron presentar diagnóstico médico de diabetes, hipertensión y cáncer, que pertenecían a la jurisdicción de los centros de salud de la Red Puente Piedra, del Ministerio de Salud de Lima; se seleccionó aleatoriamente a cuatro centros de salud de un total de doce. La selección de los sujetos se realizó siguiendo la técnica de las agujas del reloj, casa por casa, hasta completar el total de las viviendas asignadas a dichos centros de salud, lo que hace un total de 382 sujetos. La finalidad del presente estudio no es generalizar resultados de alguna población específica, ya que se trata de un primer estudio sobre este tema, por lo que se tiene un alcance exploratorio de diseño transversal.

La selección de los sujetos se realizó con los siguientes criterios de inclusión: 1) ser mayores de 18 años de edad; 2) presentar al menos una de las siguientes enfermedades: hipertensión, diabetes y/o cáncer. Si uno de los participantes presentaba dos de ellas se consideraba la enfermedad de mayor duración; y 3 ) haber presentado el diagnóstico médico como un mínimo de tres meses de antigüedad. El total de la muestra estuvo conformado por 382 personas que refirieron tener el diagnóstico médico de diabetes, hipertensión y cáncer, y ser mayores de 18 años de edad. Participaron en la aplicación de la encuesta estudiantes de Enfermería de la Universidad Cesar Vallejo (UCV), las que fueron previamente capacitadas, al igual que las docentes de práctica quienes apoyaron en el monitoreo de la aplicación de encuestas. EL instrumento original sobre «Automanejo en padecimientos crónicos. Partners in Health Scale», comprende doce ítems, con cuatro dimensiones: adherencia al tratamiento; conocimiento de la enfermedad; manejo de los efectos secundarios, y manejo de los signos y síntomas $(22,23)$. Se aplicó el mismo instrumento que fue validado previamente en población peruana, el cual presento un alpha de cronbach de 0,893 identificando tres dimensiones: conocimientos, adherencia y manejo de los síntomas. Se incluyen, en total, a los doce ítems originales del instrumento (24). Las respuestas de los doce ítems del instrumento son en escala de 0 a 8 puntos, donde más cerca a cero es menor automanejo y más cerca de ocho mejor automanejo. Para 
la interpretación de los resultados del automanejo se realizó una sumatoria de todos los ítems, creando una nueva variable donde el más cercano a cero es más deficiente, y el más cercano a 96 es el adecuado. Así mismo, se realizó la sumatoria correspondiente a cada dimensión y se convirtió cada uno de los ítems en variable ordinal de acuerdo a las siguientes categorías: de 0-2: automanejo deficiente; de 3-5 automanejo regular, y de 6-8 automanejo adecuado. Se usó el programa estadístico SPSS vs 18.
La técnica estadística utilizada para las variables continuas fue el análisis de la normalidad, una vez comprobada esta, se procedió a aplicar técnicas estadísticas correspondientes según el objetivo del estudio, utilizando la prueba de de Kruskal-Wallis para el análisis de diferencias entre grupos, ya que la prueba de normalidad de Kolmogorov Smirnou resultó significativa. Se contó con el consentimiento informado de cada participante, así como la revisión del proyecto por el comité ético de investigación de la Escuela de Enfermería de la UCV y de la autoridad del Ministerio de salud correspondiente (DISA Lima Ciudad).

\section{RESULTADOS}

Tabla 1. Distribución de la población según diagnóstico por grupos etarios

\begin{tabular}{|c|c|c|c|c|c|c|}
\hline \multirow[t]{3}{*}{ Diagnóstico } & \multicolumn{6}{|c|}{ Edades } \\
\hline & \multicolumn{2}{|c|}{ 18-39 años } & \multicolumn{2}{|c|}{ 40-59 años } & \multicolumn{2}{|c|}{60 a 90 años } \\
\hline & $\mathrm{fr}$ & $\%$ & $\mathrm{fr}$ & $\%$ & fr & $\%$ \\
\hline Hipertensión & 6 & 20,7 & 40 & 28,6 & 51 & 34,9 \\
\hline Diabetes & 8 & 27,6 & 54 & 38,6 & 49 & 33,6 \\
\hline Cáncer & 15 & 51,7 & 46 & 32,9 & 46 & 31,5 \\
\hline Total & 29 & 100 & 140 & 100 & 146 & 100 \\
\hline
\end{tabular}

* No suma el total de la muestra por haber tenido pérdida de la información en la captura de la edad

Tabla 2. Resultado del Índice general de automanejo total y por enfermedad

\begin{tabular}{|c|c|c|c|c|c|c|}
\hline \multirow[t]{2}{*}{ Índice } & \multirow[t]{2}{*}{$\mathbf{n}$} & \multirow[t]{2}{*}{ Media } & \multicolumn{2}{|c|}{$\begin{array}{c}\text { Intervalo de confianza } \\
\text { al } 95 \%\end{array}$} & \multicolumn{2}{|c|}{ Rangos } \\
\hline & & & $\begin{array}{l}\text { Límite } \\
\text { inferior }\end{array}$ & $\begin{array}{l}\text { Límite } \\
\text { superior }\end{array}$ & Mínimo & Máximo \\
\hline $\begin{array}{l}\text { Índice general de } \\
\text { automanejo }\end{array}$ & 382 & 66,1 & 64,5 & 67,6 & 9,0 & 96,0 \\
\hline Hipertensión & 134 & 64,7 & 62,2 & 67,2 & 14,0 & 94,0 \\
\hline Diabetes & 138 & 65,5 & 62,9 & 68,0 & 9,0 & 96,0 \\
\hline Cáncer & 110 & 68,4 & 65,4 & 71,4 & 15,0 & 96,0 \\
\hline
\end{tabular}

Tabla 3. Automanejo según dimensiones

\begin{tabular}{|c|c|c|c|c|c|c|c|c|c|c|}
\hline \multirow[t]{3}{*}{ Índice } & \multirow[t]{3}{*}{$\mathrm{n}$} & \multicolumn{3}{|c|}{ Conocimientos } & \multicolumn{3}{|c|}{ Adherencia } & \multicolumn{3}{|c|}{$\begin{array}{l}\text { Manejo de signos y } \\
\text { síntomas }\end{array}$} \\
\hline & & \multirow{2}{*}{ Media } & \multicolumn{2}{|c|}{ Rangos } & \multirow{2}{*}{ Media } & \multicolumn{2}{|c|}{ Rangos } & \multirow{2}{*}{ Media } & \multicolumn{2}{|c|}{ Rangos } \\
\hline & & & Min. & Max. & & Min. & Max & & Min. & Max \\
\hline $\begin{array}{l}\text { Índice general } \\
\text { de auto } \\
\text { manejo }\end{array}$ & 382 & 8,13 & 2,00 & 10,0 & 39,63 & 5,0 & 56,0 & 16,68 & 0,00 & 24,0 \\
\hline Hipertensión & 134 & 8,23 & 3,00 & 10,0 & 38,44 & 9,0 & 56,0 & 16,96 & 1,00 & 24,0 \\
\hline Diabetes & 138 & 8,28 & 2,00 & 10,0 & 38,54 & 5,0 & 56,0 & 17,15 & 2,00 & 24,0 \\
\hline Cáncer & 110 & 7,82 & 2,00 & 10,0 & 42,46 & 8,0 & 56,0 & 15,76 & 0,00 & 24,0 \\
\hline
\end{tabular}


Tabla 4. Diferencias del auto manejo entre grupos:prueba de Kruskal-Wallis

\begin{tabular}{lllcc}
\hline \multicolumn{1}{c}{ Dimensiones } & Diagnóstico & N & Rango promedio & Sig \\
\hline \multirow{2}{*}{ Índice general } & Hipertensión & 134 & 181,87 & Chi $^{2}: 3,423$ \\
& Diabetes & 138 & 188,19 & Gl 2 \\
Cáncer & 110 & 207,39 & p: 0,181 \\
Total & 382 & & \\
Conocimientos & Hipertensión & 134 & 192,58 & Chi $^{2}: 2,898$ \\
& Cáncer & 138 & 201,22 & Gl 2 \\
Total & 110 & 177,99 & p: 0,235 \\
Adherencia & Hipertensión & 134 & & \\
& Diabetes & 138 & 176,65 & Chi $^{2}: 12,030$ \\
& Cáncer & 110 & 181,51 & Gl 2 \\
Total & 382 & 222,12 & p: 0,002 \\
Míntomas & Hipertensión & 134 & & Chi ${ }^{2}: 8,248$ \\
& Diabetes & 138 & 199,98 & Gl 2 \\
\hline & Cáncer & 110 & 203,45 & p: 0,016 \\
& Total & 382 & 166,17 & \\
\hline
\end{tabular}

* Variable de agrupación: Diagnóstico

Tabla 3. Automanejo según dimensiones

\begin{tabular}{lcccc}
\hline Automanejo & $\begin{array}{c}\text { Femenino } \\
\text { fr }\end{array}$ & \% & $\begin{array}{c}\text { sexo } \\
\text { masculino } \\
\text { fr }\end{array}$ & \% \\
\hline Regular/Deficiente & 114 & 51.4 & 46 & 37.7 \\
Adecuado & 108 & 48.6 & 76 & 62.3 \\
Total & 222 & 100.0 & 122 & 100.0 \\
\hline
\end{tabular}

* Chi2 : p: 0.01

* No suma el total de la muestra por haber tenido pérdida de la información en la captura del sexo

Los resultados representan al $100 \%$ de las familias pertenecientes a comunidades asignadas a centros de salud del Ministerio de Salud incluidas en el estudio, la tasa de no respuesta fue de $5 \%$, por no encontrar a nadie en las viviendas. La población presentó una media de edad de 57 años, con rangos de 18 a 90 años de edad. El 87\% refiere estar actualmente con tratamiento. El 32\% (122) son de sexo masculino y el 58\% (222) de sexo femenino; en 41 sujetos no se registró el sexo. Su distribución, según en- fermedad, se presenta en la Tabla 1, se encontró mayores porcentajes de diagnóstico de cáncer en la población de 18-39 años de edad en comparación con los grupos mayores a 40 años de edad (52 vs. $32 \%$ ).

Los resultados en el automanejo, de un rango de 0 a 96 , presentaron una media de 66, con rangos de 9 a 96. El $2,1 \%$ (8) presento un automanejo deficiente; 40,8\% (157) automanejo regular, y 57,1\% (220) adecuado. Las medias 
por enfermedad mostraron, para el caso de hipertensión, una media de 64; para diabetes, una media de 65, y para cáncer una media de 68 (Tabla 2). Los resultados en la dimensión de conocimiento, el grupo con cáncer mostró la media más baja (7 vs. 8) en comparación con el índice general y las otras dos enfermedades; en la dimensión de adherencia, la media más alta se presentó en el grupo con cáncer (42), a diferencia de los otros grupos que fue de 38; en la dimensión de manejo de signos y síntomas, la media más alta la alcanzó el grupo con diabetes (17) a diferencia de los otros grupos que fue de 16 y 15 (Tabla $3)$.

En el análisis de diferencias entre las enfermedades y el índice general, los resultados de la prueba de KruskalWallis mostraron diferencias significativas entre la dimensión de adherencias y la dimensión del manejo de signos y síntomas con <0,05 (Tabla 4). En cuanto el automanejo según sexo, se encontró asociación significativa, $\left(\mathrm{chi}^{2}\right.$ $\mathrm{r}<0,05$ ); en el grupo de regular y deficiente el sexo femenino presentó el $51 \%$ (114), a diferencia del sexo masculino que fue el $37,7 \%$ (46) (Tabla 5).

\section{DISCUSIÓN}

Los resultados demográficos de la población estudiada muestran un rango bastante amplio en las edades que refieren presentar una de estas tres enfermedades crónicas ( hipertensión, diabetes y cáncer) desde 18 a 90 años de edad, aspecto que llama la atención pues, en la literatura revisada hasta el momento, se reporta edades con mayor prevalencia de enfermedades crónicas a partir de los 40 a más años de edad $(6,7,25)$; así mismo, cabe señalar que el mayor porcentaje reportado con diagnóstico de cáncer es en el grupo de 18 a 39 años de edad en comparación con los de 40 a más años, aunque el presente estudio no tuvo por finalidad medir prevalencias de enfermedades crónicas, estos resultados podrían estar sugiriendo realizar investigaciones de estudio de prevalencia en jóvenes.

Con relación al comportamiento en el manejo de su enfermedad, y al ser este estudio el primero en realizarse en población peruana, solo se puede hacer referencias con otros estudios similares relacionados con conocimiento en el autocuidado en la diabetes. En el presente estudio se encuentra que solo el 57\% reportó un automanejo adecuado, con un media de 66, estos resultados indican que esta población presenta un automanejo de su enfermedad muy poco favorable, lo cual evidencia un problema muy importante que abordar, pues ello estaría indicando que estamos frente a personas que, teniendo una enfermedad crónica como las incluidas en el presente estudio : hipertensión, diabetes y cáncer, no presentan conocimientos adecuados sobre su salud y la enfermedad que padecen; no identifican signos y síntomas de alarma, ni toman decisiones oportunas cuando estos signos de alarma se presentan; no están manejando las consecuencias físicas, sicologías y sociales derivadas de la enfermedad que padecen, así como no presentan una adherencia al tratamiento de manera apropiada, ni presentan una adecuada interrelación con el equipo de salud del servicio donde acuden. Esta situación puede repercutir en su calidad de vida, presentar complicaciones al presentarse mayor uso de los servicios de salud y mayor gasto que esto origina a ellos, sus familias y el sistema de salud. Aunque no es posible comparar con otros resultados similares en el Perú, algunos de ellos como es el de conocimiento, son corroborados por otros estudios realizados con personas con diabetes, donde el nivel de conocimiento sobre el autocuidado alto o adecuado oscila entre $23 ; 15$ y $5 \%$ respectivamente $(8-11)$.

Los resultados también evidencian diferencias de manera estadística en las dimensiones de adherencia y manejo de signos y síntomas. En la dimensión de adherencia es el grupo con cáncer quien presenta una media mayor con relación a los otros grupos, y en la dimensión de manejo de signos y síntomas es el grupo de diabetes quien presenta una media mayor en comparación con los otros grupos; estos resultados sugirieren la presencia de necesidades diferentes por abordar, según sea la enfermedad; así mismo, esta diferencia podría estar también demostrando que una persona con cáncer tiene mejor entendimiento de la importancia del cumplir con el tratamiento y las citas, pero no es así en el manejo de sus signos y síntomas como consecuencia de la enfermedad en aspectos físico, sicológicos y sociales. Aspecto diferente podríamos asumir en el grupo de personas con diabetes, donde presentan medias más altas en el manejo de signos y síntomas más no en adherencia al tratamiento y citas. En el grupo de personas con hipertensión, se encuentran deficiencias en las tres dimensiones. Por otro lado, es importante destacar las diferencias encontradas en el automanejo según sexo, cuyos resultados sugieren la necesidad de seguir investigando este comportamiento con una perspectiva de género.

Está demostrado los beneficios de un adecuado automanejo en personas con enfermedades crónicas, entendiendo esto no solo en un adecuado conocimiento de la enfermedad, sino además en un adecuado manejo en la toma de decisiones oportunas, de acuerdo a los signos y síntomas presentados, adherencia al tratamiento, y manejo adecuado 
de los aspectos sicológicos y sociales que podrían ser afectados por la presencia de una enfermedad crónica (20 22), por lo que estos resultados nos permiten por primera vez tener una línea basal de donde estamos con respecto al automanejo en enfermedades crónicas, resultado no alentador.

Es importante señalar que la principal limitación del presente estudio es el diseño muestral, pues al ser una muestra por conveniencia, no permite generalizar los resultados a poblaciones similares. Sin embargo, consideramos que los resultados son confiables, ya que se ha tenido extremo cuidado con validar previamente el instrumento (24) en poblaciones similares donde se ha aplicado el estudio, así como el proceso de selección de los sujetos, recolección y captura de la información.

El presente estudio, permite concluir que hay una gran necesidad de seguir realizando estudios que admitan conocer el comportamiento de las personas con enfermedades crónicas, referente a su manejo y cuidados; así mismo, reorientar y fortalecer los programas educativos destinados a promover el automanejo bajo una perspectiva colaborativa entre la persona con la enfermedad y los profesionales de salud.

Por otro lado, los resultados sugieren hipótesis de estudio para próximas investigaciones tales como: estudios de prevalencia en grupos de jóvenes (18 a 39 años de edad), así como investigaciones aplicativas que fortalezcan el automanejo en personas con enfermedades crónicas, con una perspectiva de género.

Agradecimiento: el presente estudio recibió apoyo financiero de la Escuela de Enfermería de la Universidad Cesar Vallejo, Lima Norte. Participaron sus docentes y estudiantes de pregrado de Enfermería. Así mismo, se agradece la colaboración de los profesionales de Enfermería de los centros de salud por su valiosa colaboración.

\section{REFERENCIAS BIBLIOGRÁFICAS}

1. United Health, National Heart Lung Blood Institute Centers of Excellence, Cerqueira MT, Cravioto A, Dianis N, Ghannem H, et al. Global response to noncommunicable disease. BMJ. 2011; 342-d3823.doi: 10.1136/bjm.d5328

2. Sridhar D, Morrison J, Piot P. Getting the Politics Right for the September 2011 UN High-Level Meeting on Noncommunicable Diseases. Washington DC: Center for Strategic and International Studies; 2011.
3. World Health Organization. Preventing chronic diseases: a vital investment. WHO Global Report. Geneva: World Health Organization; 2005.

4. Organização Mundial da Saúde. Cuidados inovadores para condições crônicas: componentes estruturais de ação. Relatório Mundial. Brasília: OMS; 2003.

5. Amador-Díaz MB, Márquez-Celedonio FG y SabidoSighler AS. Factores asociados al auto-cuidado de la salud en pacientes diabéticos tipo 2. Asociación Latinoamericana de Profesores de Medicina Familiar. Mexico, 2007; 9 (2): 99-107

6. Miranda J, Wells J y Smeeth L. Transiciones en contexto: Hallazgos vinculados a migración ruralurbana y enfermedades no transmisibles en Perú. Rev. perú. med. exp. salud pública. [Internet] 2012. Julset [citado 27 Mayo 2013]; 29 (3): 366-372. Disponible en: <http://www.scielo.org.pe/ scielo.php?script $=$ sci_arttext \&pid=S 1726 $46342012000300012 \& \operatorname{lng}=e s \& n r m=i s o>$. ISSN 17264634.

7. Ministerio de Salud. Dirección General de Salud de las Personas.Estrategia Sanitaria Nacional de Prevención y Control de Daños No Transmisibles. «Situación actual de las enfermedades no trasmisibles en el país y prioridades para los siguientes años». Noviembre, 2010.

8. Pace A, Ochoa-Vigo K, Larcher M, Morais A. El conocimiento sobre diabetes mellitus en el proceso de autocuidado. Rev Latino-am Enfermagem, 2006; 14(5)

9. Murata GH, Shah JH, Adam KD, Wendel CS, Bokhari SU, Solvas PA, et al. Factors affecting diabetes knowledge in type 2 diabetic veterans. Diabetologia, 2003; 46: 1176-8.

10. Cabrera-Morón R, Motta-Quijandría I, RodríguezRobladillo C, Velásquez-Carranza D. Nivel de conocimiento sobre autocuidado en la prevención de complicaciones diabéticas en usuarios del Policlínico Chincha - EsSalud. Ica; 2009.

11. Baca Martínez, B. y Col. Nivel de conocimientos del diabético sobre su autocuidado Hospital General Nro1 de Culiacán Sinaloa; 2008 [en línea]. México: [citado 10 de junio del 2009]. Disponible en: http:// revistas.um.es/eglobal/article/ViewFile/14691/14171

12. Corbacho A y Palacios G. Relación entre el nivel de conocimiento y la práctica de estilos de vida del paciente con diabetes mellitus en el Hospital Nacional Arzobispo Loayza. [Tesis de Licenciatura]. Lima: Universidad Peruana Cayetano Heredia; 2008.

13. Doak CC, Doak LG, Root JH. The literacy problem. En:Teaching Patients with Low Literacy Skills. 2. ${ }^{a}$ ed. Philadelphia: J.B. Lippincott Co; 1996. p. 1-9. 
14. Bodenheimer T, Lorig K, Holman H, Grumbach K. Patient self-management of chronic disease in primary care. JAMA, 2002; 288(19): 2469-75.

15. Lawn S, Battersby M, Harvey P, Pols R, Ackland A. A behavioural therapy approach to selfmanagement:the Flinders Program. DIABETES VOICE, 2009; 54

16. Ryan P y Sawin KJ. The individual and family selfmanagement theory: background and perspectives on context, process, and outcomes. Nurs Outlook, 2009; 57(4): 217-225.

17. Lorig K, Halsted R .Self-Management Education: History, Definition, Outcomes, and Mechanisms. SelfManagement Education. Volume 26, Number 1, 2003

18. Corbin J y Strauss A. Unending Work and Care: Managing Chronic Illness at Home. San Francisco: Jossey-Bass, 1988.

19. Lorig K y Holman H. Self-management education: history, definition, outcomes, and mechanisms. Ann Behav Med, 2003; 26(1):1-7.

20. Lorig KR, Ritter PL y González VM. Hispanic chronic disease self-management: a randomized communitybased outcome trial. Nurs Res, 2003; 52(6): 361-9.

21. Thoesen M y Newton K. Supporting Selfmanagement in Patients with Chronic Illness.American Family Physician, 2005; 72 (8).

22. Petkov J, Harvey P y Battersby M. The internal consistency and construct validity of the partners in health scale: validation of a patient rated chronic condition self-management measure. Qual Life Res, 2010; (19): 1079-1085
23. Battersby M, Ask A, Reece M, Markwick M y Collins J. The Partners in Health scale: The development and psychometric properties of a generic assessment scale for chronic condition self-management. Australian Journal of Primary Health, 2003; 9

24. Peñarreita I, Vergel O, Lezama S, Rivero R, Taipe J y Bejarano L. Validación de un instrumento para evaluar el auto manejo en enfermedades crónicos en el primer nivel de atención, Lima - Perú. Aceptado para su publicación en la revista Enfermería Científica Colegio Nacional de Enfermería Peruana. Lima, 2013.

25. Rosas A, Lama G, Llanos-Zavalaga F y Dunstan J. Prevalencia de obesidad e hipercolesterolemia en trabajadores de una institución estatal de Lima - Perú. Revista Peru Med Exp Salud Publica, 2002; (2) 19

\section{Correspondencia}

María Isabel Peñarrieta

Correo electrónico: decordova.maria.isabel@gmail.com

pcordoba@uat.edu.mx

Forma de citar este artículo: Peñarrieta-De Córdova M, VergelCamacho S, Lezama-Vigo S, Rivero- Álvarez R, Taipe-Cancho J, Borda-Olivas H. El automanejo de enfermedades crónicas: población de una jurisdicción de centros de salud. Rev enferm Herediana. 2013;6(1):42-49. 